\title{
Safety and efficacy of endoscopic submucosal dissection for metachronous early cancer or precancerous lesions emerging at the anastomotic site after curative surgical resection of colorectal cancer
}

\author{
Zhihao Chen ${ }^{1}$, Lizhou Dou ${ }^{1}$, Yueming Zhang ${ }^{1}$, Shun $\mathrm{He}^{1}$, Yong Liu ${ }^{1}$, Huizi Lei ${ }^{2}$, Guiqi Wang ${ }^{1}$ \\ ${ }^{1}$ Department of Endoscopy, National Cancer Center/National Clinical Research Center for Cancer/Cancer Hospital, Chinese Academy of Medical \\ Science and Peking Union Medical College, Beijing, China; ${ }^{2}$ Department of Pathology, National Cancer Center/National Clinical Research Center \\ for Cancer/Cancer Hospital, Chinese Academy of Medical Science and Peking Union Medical College, Beijing, China \\ Contributions: (I) Conception and design: All authors; (II) Administrative support: G Wang; (III) Provision of study materials or patients: L Dou, Y \\ Zhang, S He, Y Liu, H Lei, G Wang; (IV) Collection and assembly of data: Z Chen; (V) Data analysis and interpretation: Z Chen; (VI) Manuscript \\ writing: All authors; (VII) Final approval of manuscript: All authors. \\ Correspondence to: Guiqi Wang. Director of Endoscopic Department, Department of Endoscopy, National Cancer Center/National Clinical Research \\ Center for Cancer/Cancer Hospital, Chinese Academy of Medical Science and Peking Union Medical College, 17 Panjiayuannanli, Beijing 100021, \\ China. Email: wang_guiqi@126.com; wangguiq@126.com.
}

\begin{abstract}
Background: The incidence of metachronous early cancer or precancerous lesions (MECPL) emerging at the anastomotic site (AS) after curative surgical resection of colorectal cancer (CRC) is so low that few study have been conducted to explore the clinical characteristics, diagnosis and treatment of these lesions. Endoscopic submucosal dissection (ESD) is technically difficult for these lesions because of the presence of severe fibrosis and AS. The aim of this study was to explore the safety and efficacy of ESD for MECPL emerging at the AS after curative surgical resection of CRC.

Methods: The data used in the analysis were retrospectively collected from CICAMS in Beijing China between January 2013 and May 2019 and from all the patients who underwent ESD for MECPL emerging at the AS after curative surgical resection of CRC. The rates of en bloc resection (ER), complete resection (CR), curative resection (CuR) and incidence of complications were analyzed by SPSS software.

Results: A total of 11 patients were included. The rates of ER, CR and CuR were 63.6\%, 54.5\% and $54.5 \%$, respectively. No additional surgery was performed, and no recurrences were found. Bleeding occurred in only one case and there was no perforation after the operation.

Conclusions: Overall, ESD is safe and effective in the treatment of MECPL emerging at the AS after curative surgical resection of CRC. Especially for patients with anastomotic recurrence close to anal margin, this method can avoid the risks of reoperation and improve the rate of anal preservation.
\end{abstract}

Keywords: Colorectal cancer (CRC); anastomotic sites (AS); early cancer; precancerous lesions; endoscopic submucosal dissection (ESD)

Submitted Mar 07, 2020. Accepted for publication Aug 27, 2020.

doi: 10.21037/atm-20-2064

View this article at: http://dx.doi.org/10.21037/atm-20-2064

(c) Annals of Translational Medicine. All rights reserved. 


\section{Introduction}

Colorectal cancer (CRC) is the fourth most common neoplasia and the second leading cause of death in the world $(1,2)$, for which surgery is the mainstay of treatment. Compared to the general population, patients undergoing surgery for CRC are more likely to face an increasing risk of developing metachronous neoplasia in the remaining colorectum $(3,4)$.

In clinical practice, we encountered the following situation: a small number of CRC patients completely cured by standard treatment through curative surgical resection with negative resection margins, several years later, were found to have metachronous early cancer or precancerous lesions (MECPL) emerging at the anastomotic site (AS) by colonoscopy that did not originate from the primary tumor. Salvage operation is the primary treatment for this situation (5). However, salvage operation means a heavy burden for these patients, both physically and economically, especially for those with low anastomotic lesions $(6,7)$. Due to the low incidence of MECPL emerging at AS, only three studies $(5,8,9)$ with a few cases have been performed to explore the feasibility of endoscopic submucosal dissection (ESD) for MECPL emerging at the AS after curative surgical resection of CRC. The clinical characteristics, diagnosis and treatment of these lesions still remain unclear. Therefore, this study will explore the safety and efficacy of ESD for MECPL emerging at the AS after curative surgical resection of CRC. We present the following article in accordance with the STROBE reporting checklist (available at http://dx.doi.org/10.21037/atm-20-2064).

\section{Methods}

\section{Patient characteristics and indications for ESD}

Patients who underwent ESD for MECPL emerging at the AS after curative surgical resection of CRC at the Cancer Institute and Hospital, Chinese Academy of Medical Sciences (CICAMS), Beijing, China between January 2014 and May 2019 were retrospectively reviewed. Abdominopelvic enhanced computed tomography (CT) scan was conducted in all included patients to assess the presence of regional lymph node involvement and distant metastasis. A variety of endoscopic techniques such as white light endoscopy, chromoendoscopy, narrow-band imaging (NBI), magnifying endoscopy and ultrasound endoscopy were used to evaluate whether the lesions had indications for endoscopic treatment (10). The indication criteria for endoscopic treatment in this study followed the guidelines for ESD of early colorectal tumors: biopsy-proven low/ high-grade intraepithelial neoplasia or adenocarcinoma with invasion less than $1,000 \mu \mathrm{m}$ into the submucosa (11). Patients with positive resection margins including the circumferential resection margin (CRM) in the final pathological examination (12) were not included in this study. In this way, we excluded those cases of residual lesions or recurrence of the original tumor. The study was conducted in accordance with the Declaration of Helsinki (as revised in 2013). The surgical procedures were approved by the Department of Endoscopy at the National Cancer Center/Cancer Hospital, Chinese Academy of Medical Sciences (CICAMS), and the Ethics Committee of the National Cancer Center/Cancer Hospital, Chinese Academy of Medical Science and Peking Union Medical College (Approval Number: 18-002/1466). Written informed consent was obtained from the patients for the surgery and for publication of this cohort study and any accompanying images.

\section{ESD procedures}

All procedures were performed by experienced endoscopists (who have performed more than 1,000 ESD procedures) at CICAMS with a dual knife (KD-650Q; Olympus Optical, Tokyo, Japan) and a single-channel endoscope (PCF-Q260JI, GIF-Q260J; Olympus) with a transparent hood (D-20111804; Olympus) attached to its tip. In brief, we marked the normal mucosa that surrounded the lesion at least $5 \mathrm{~mm}$ away from the tumor with the dual knife. After the injection of a saline solution with epinephrine $(0.025 \mathrm{mg} / \mathrm{mL})$ into the submucosa, an initial cut, also called a precut, was made with a standard needle knife on the oral side of the tumor, and then a circumferential mucosal incision was made around the tumor. All of the anastomotic nails (ANs) needed to be removed by peeling off the tissue surrounding the ANs (see in Figure 1). The submucosal injection was repeated during the procedure if necessary, and endoscopic hemostasis was achieved. The tumor was then completely removed by submucosal dissection. After lesion removal, preventive coagulation was performed for all visibly exposed vessels with hot biopsy forceps. Then, endoscopic clips were used for prophylactic closure of defects at the AS. A high-frequency electrosurgical current generator (Erbotom VIO 300D; ERBE, Tübingen, Germany) was used during 


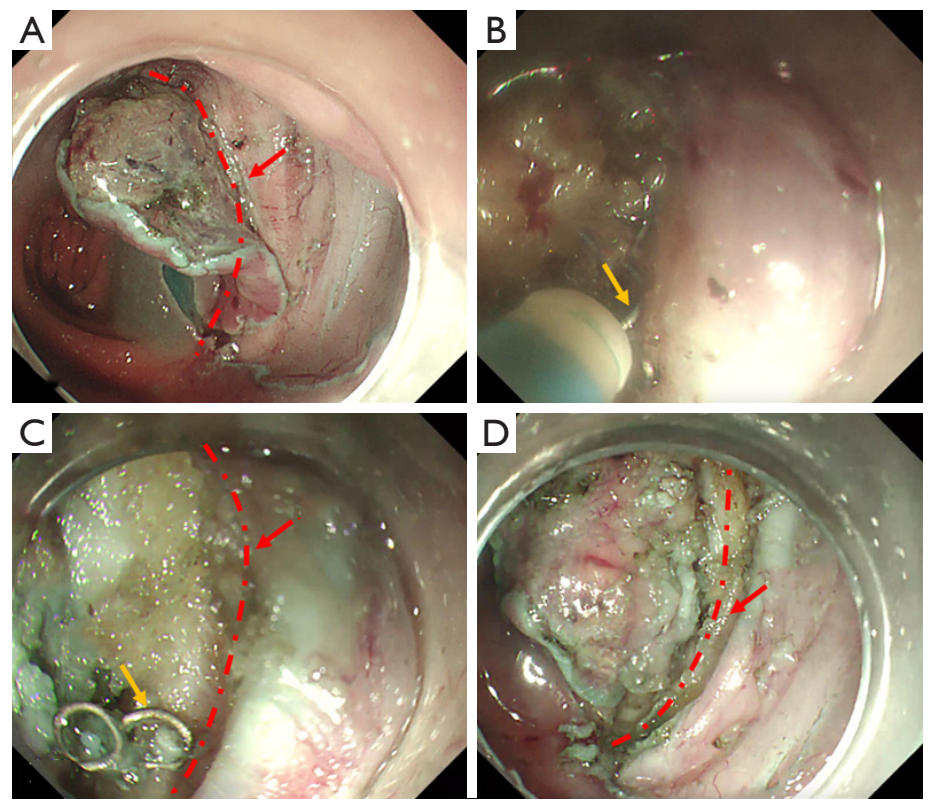

Figure 1 Endoscopic procedure for peeling the lesions and removing of anastomotic nails (ANs). (A) Endoscopic submucosal dissection of the lesion on the oral side of the anastomosis site (AS); (B) the dual knife was placed in contact with the AN when peeling off tissues around the AS, which integrated the nail and electrical knife by means of the electrical conduction effect; (C) the AN was gently and successfully pulled out by the dual knife; (D) all ANs at the AS were pulled out.

the marking, mucosal incision, submucosal dissection, and hemostasis.

\section{Pathological assessment of the resected specimens}

The endoscopically resected specimens were serially sectioned in millimeter intervals. The histological classification of adenocarcinoma was performed according to the Vienna classification of colonic epithelial neoplasms (13). The depth of invasion, presence of lymphatic and/or vascular invasion, margins, and tumor differentiation were evaluated. An en bloc resection (ER) referred to the endoscopic resection of the tissue in one piece, whereas complete resection (CR) was histologically defined as ER of the tumor with horizontal margins (HMs) and vertical margins (VMs) free of tumor tissue (11). In addition, we also defined histologically curative resection $(\mathrm{CuR})$ based on the following conditions: ER, HM (-), VM (-), lymphatic involvement (-), vessel involvement $(-)$, and $<1,000 \mu \mathrm{m}$ from the muscularis mucosa (11).

\section{Follow-up}

After ESD, the patients who underwent curative resection had colonoscopy twice, one 3 months later and the other 12 months later, and an annual surveillance was performed thereafter. For the patients who underwent noncurative resection, surgical resection was carried out following a routine protocol. The patients with noncurative resection who refused surgical resection underwent endoscopy and/or endoscopic ultrasonography (EUS) in addition to CT every 3 to 6 months.

\section{Statistical analysis}

The baseline characteristics of the patients were expressed as the mean \pm standard deviation (SD) or range (median). All statistical analyses were performed using SPSS 24.0 (SPSS, Chicago, IL, USA).

\section{Results}

\section{Baseline and clinicopathological characteristics}

Among 2,385 cases of colorectal lesions treated by ESD in our endoscopic center, only 11 patients with MECPL emerging at the AS, with a mean age of $61.9 \pm 11.5$ years old and a male/female ratio of $9 / 2(81.8 \%: 18.2 \%)$, were 


\section{Page 4 of 8}

Table 1 Clinicopathologic characteristics of the study patient $(\mathrm{n}=11)$

\begin{tabular}{lc}
\hline Characteristics & Data \\
\hline Age (years) & \\
Mean \pm SD & $61.9 \pm 11.5$ \\
Sex ratio (Male:Female) & $9: 2$ \\
Emerging time (months) & \\
Min-max (median) & $36-252(147.0)$ \\
Tumor size of emerging lesions (mm) & \\
Min-max (median) & $7-31(20.0)$ \\
Distance from the lesion to the anal margin & \\
(cm) & \\
$\leq 7$ cm & $6(54.5 \%)$ \\
$>7$ cm and $\leq 15$ cm & $3(27.3 \%)$ \\
$>15$ cm & $2(18.2 \%)$ \\
Min-max (median) & $3-60(6.0)$ \\
History of chemotherapy & \\
No & $6(54.5 \%)$ \\
Yes & $5(45.5 \%)$ \\
Nistory of radiotherapy & $5(45.5 \%)$ \\
\hline
\end{tabular}

treated by ESD between January 2013 and May 2019 at the CICAMS in Beijing, China. All patient characteristics and operation details for the entire research are provided in Table 1 and Table 2. The diameter of the emerging lesions ranged from 7 to 31 (median 20) $\mathrm{mm}$. The time until MECPL emerged ranged from 36 to 252 (median 147.0) months. The operation time ranged from 40 to 262 (median 48.0) minutes, and the average hospital stay was $6.0 \pm 1.9$ days. In addition, detailed information of each patient and the pathologic characteristics of the primary tumor are presented in the Tables S1-S4.

\section{Short-term outcomes}

The diameter of the resected specimens ranged from 12 to 36 (median 30 ) $\mathrm{mm}$. These lesions were evaluated by histopathologic examinations, and 2 of them were found to have both positive HMs and positive VMs. No lymphatic or vessel invasion was found among any of the 11 specimens. According to the pathological results, the ER was achieved

\section{Chen et al. ESD for MECPL emerging at the AS after CRC surgery}

Table 2 Operation details of endoscopic submucosal dissection

\begin{tabular}{lc}
\hline Variables & Data \\
\hline Operation duration (minutes) & \\
Min-max (median) & $40-262(48.0)$ \\
Hospital time (d) & $6.0 \pm 1.9$ \\
Mean \pm SD & \\
Complications, No. (\%) & $1(9.1 \%)$ \\
Postoperative bleeding & $0(0 \%)$ \\
Perforation & \\
Follow-up time (months) & $6-61(14.0)$ \\
Min-max (median)
\end{tabular}

for 7 lesions (63.6\%), and both CR and CuR were achieved for 6 lesions $(54.5 \%)$. Five patients with noncurative resection refused surgical resection and underwent endoscopic follow-up evaluations on a regular basis. The pathological characteristics of ESD are shown in Table 3. Bleeding $(\mathrm{n}=1,9.1 \%)$ occurred in 1 patient $(9.1 \%)$ and was managed endoscopically without any serious consequences. No microperforation was observed.

\section{Long-term outcomes}

During the follow-up period (range, 6-61 months, median 14 months), we did not observe local or distant recurrences in any of the patients with curative resection or in any of the 5 patients with noncurative resection who rejected additional surgery.

\section{Discussion}

Although three papers $(5,8,9)$ have reported the feasibility of endoscopic treatment for MECPL emerging at the AS, no clear definition of the indications exists. In our study, we provided a clear definition of the concept of MECPL emerging at the AS, and only metachronous colorectal lesions with a negligible risk of lymph node metastasis emerging at the AS met the indications, which was inconsistent with the nature and depth of invasion of the original tumor. In all included cases, the CRC tumors, with presented with different pathologic types and different tumor stages, were completely removed by standard treatment through curative surgical resection of $\mathrm{CRC}$ with negative resection margins. Another 3 lesions 
Table 3 Pathological results of endoscopic submucosal dissection

\begin{tabular}{|c|c|}
\hline Variables & Data \\
\hline \multicolumn{2}{|c|}{ Size of resected specimen (mm) } \\
\hline Min-max (median) & $12-36(30.0)$ \\
\hline \multicolumn{2}{|l|}{ Depth of invasion } \\
\hline LGIN/HGIN & $10(90.9 \%)$ \\
\hline M & $1(9.1 \%)$ \\
\hline $\mathrm{SM}(\leq 1,000 \mu \mathrm{m})$ & $0(0 \%)$ \\
\hline $\mathrm{SM}(>1,000 \mu \mathrm{m})$ & $0(0 \%)$ \\
\hline \multicolumn{2}{|l|}{ Lymphatic invasion } \\
\hline Negative & $11(100 \%)$ \\
\hline Positive & $0(0 \%)$ \\
\hline \multicolumn{2}{|l|}{ Vessel invasion } \\
\hline Negative & $11(100 \%)$ \\
\hline Positive & $0(0 \%)$ \\
\hline \multicolumn{2}{|l|}{ Horizontal margin } \\
\hline Negative & $9(81.8 \%)$ \\
\hline Positive & $2(18.2 \%)$ \\
\hline \multicolumn{2}{|l|}{ Vertical margin } \\
\hline Negative & 9 (81.8\%) \\
\hline Positive & $2(18.2 \%)$ \\
\hline \multicolumn{2}{|l|}{ Predominant type } \\
\hline LGIN & $8(72.7 \%)$ \\
\hline HGIN & $2(18.2 \%)$ \\
\hline WDA & $1(9.1 \%)$ \\
\hline MDA & $0(0 \%)$ \\
\hline PDA & $0(0 \%)$ \\
\hline \multicolumn{2}{|c|}{ Results of resection, No. (\%) } \\
\hline ER & $7(63.6 \%)$ \\
\hline CR & $6(54.5 \%)$ \\
\hline CuR & $6(54.5 \%)$ \\
\hline
\end{tabular}

LGIN, low grade intraepithelial neoplasia; HGIN, high grade intraepithelial neoplasia; M, mucosa; SM, submucosa; WDA, well-differentiated adenocarcinoma; MDA, moderately differentiated adenocarcinoma; PDA, poorly differentiated adenocarcinoma; ER, en-bloc resection; CR, completed resection; CuR, curative resection. at the AS in our center found to emerge in a short time after CRC surgery were excluded from our study, because these cases might be the residual lesions or recurrences of the original tumor. The time until MECPL emerged at the AS varied from 3 to 21 years, which confirms that regular colonoscopy is an indispensable examination during followup. In addition, the preoperative endoscopic diagnosis of all MECPL emerging at the AS was based on the Kudo's pit pattern classification and the JNET classification, and the nature and depth of the lesions evaluated by endoscopy were basically consistent with postoperative pathology. Therefore, the current diagnostic techniques and theoretical systems for evaluating the nature and depth of colorectal tumor may be applicable to MECPL emerging at the AS $(14,15)$.

Previous data (16) confirmed that the incidence of delayed bleeding after endoscopic treatment for early CRC is $6.3 \%$, while that of perforation is $5.4 \%$. However, only one patient $(9.1 \%)$ had postoperative bleeding and was successfully stopped by endoscopic electrocoagulation. A meta-analysis confirmed that there was no significant difference on postoperative bleeding between suture line and non-suture line lesions (17). Similarly, in the study conducted by Maehata et al. (5), none of the 11 patients had postoperative perforations, and the reason may be that the surrounding tissues proliferate and adhere to envelope the AS and its surrounding intestinal wall during the healing process after curative surgical resection of CRC. As a result, the AS and its surrounding intestinal wall become thicker and less prone to perforation. In addition, carbon dioxide insufflation was used during the ESD procedures because it was rapidly absorbed and its prophylactic effect may therefore be better than that of air insufflation in the event of a perforation (18-20). Hence, these results demonstrate the safety of ESD for MECPL emerging at the AS after curative surgical resection of CRC.

Compared to those in a recent study (5), the ER rate in our study was significantly lower (63.6\% vs. $90.9 \%)$. However, the rates of VM (+), HM (+), CR and CuR were similar between the two studies, and the following explanations can be concluded. In five cases of noncurative resection, 4 lesions were resected in pieces, and another lesion was found to have both positive HMs and positive VMs. In addition, four of these five patients were treated in 


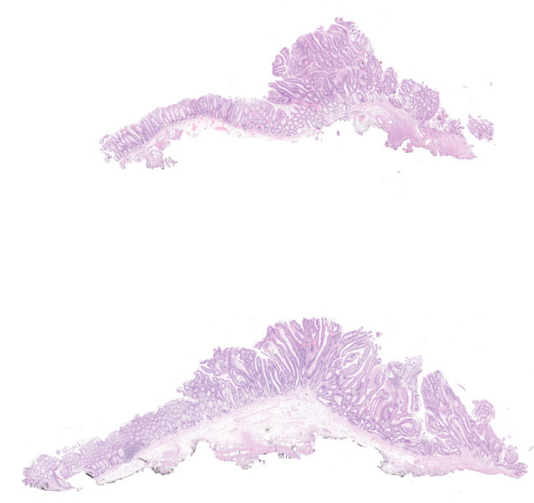

Figure 2 A comprehensive pathological picture of a resected sample with HE staining $(\times 5)$.

the early stages of using ESD to treat MECPL emerging at the AS. Without subjective factors considered, some difficulties that we encountered during the operations should not be ignored. First, the severity of submucosal adhesion of the AS scars led to the unclear demarcations of the intestinal tract at all levels (see in Figure 2) $(5,8,9,21,22)$. Moreover, the mucosa was swollen and brittle from previous radiotherapy or chemotherapy, which was not conducive to dissection. Therefore, in order to maintain the integrity of the tissue, sometimes we carefully peeled slightly deeper into the superficial intrinsic muscularis during the dissecting process. In addition, countertraction devices and the pocketcreation method have been reported to help overcome these technical difficulties $(23,24)$. The efficient strategy described by Maehata et al. (5) was to initially dissect into submucosal tissue without fibrosis, and after reaching the AS, estimate and peel along the left and right Petz line or muscular layer lines. Although the CuR rate was low and all 5 patients with noncurative resection refused surgical resection, local or distant recurrences were not observed in any of the included patients during the regular colonoscopy and CT follow-up evaluations. Thus, these results confirm that ESD is effective in the treatment of MECPL emerging at the AS after curative surgical resection of CRC.

The most important step of these operations is to pull out the ANs. Unlike forcibly extracting the ANs using alligators or forceps in ESD for early gastric remnant cancer or colorectal MECPL emerging at the AS reported in the previous studies $(8,9,18,25,26)$, the key and innovative point of our method is to integrate the nail and electrical knife by means of the electrical conduction effect (see in Figure 1B). Then, the submucosal tissue can be stripped around the
ANs step by step, and finally, the ANs can be gently and successfully pulled out (see in Figure 1C). This method can reduce the incidence of perforation and may explain why our perforation rate is much lower than that of ESD for early gastric remnant cancer emerging at the AS $(17,27)$.

Our study had several limitations that should be noted. First, our analysis is retrospective and nonrandomized and was conducted at only one center. In addition, some patients who had such lesions underwent surgery instead of ESD; thus, potential selection and referral biases cannot be excluded. Second, only a small number of cases are included in our study due to the low incidence of MECPL emerging at the AS, hence the risk factors associated with low ER, $\mathrm{CR}$, and $\mathrm{CuR}$ rates cannot be explored. Finally, the followup time (range, 6-61 months; median 14 months) is so short that some outcomes cannot be examined during this period. However, our study is the first to define metachronous lesions at the AS after CRC surgery in a more detailed way, which can help establish more accurate criteria for the indications for ESD of MECPL emerging at the AS. In our study, $81.8 \%$ of the enrolled patients had lesions at or within $15 \mathrm{~cm}$ from the anal margin. Thus, the significance of this operation is that it can reduce the probability of reoperation, especially in those cases where the AS is close to the anal margin, and increase the rate of anal preservation, thereby alleviating the trauma to the patients and improving the quality of patients' life.

In conclusion, the use of ESD may be safe and effective in the treatment of MECPL emerging at the AS after curative surgical resection of CRC. With the increase in the early diagnosis rate of CRC and the decrease in mortality after curative surgical resection (2), more MECPL emerging 
at the AS may be found during regular colonoscopy in the future. Therefore, additional prospective multicenter studies with a larger number of cases are required to confirm the safety and clinical efficacy of ESD for MECPL emerging at the AS.

\section{Acknowledgments}

We gratefully acknowledge the work of past and present members of department of Endoscopy/Pathology, National Cancer Center/National Clinical Research Center for Cancer/Cancer Hospital, Chinese Academy of Medical Science and Peking Union Medical College, Beijing, China. Besides, we also appreciate the medical writing service company (American Journal Experts, AJE, https://www.aje. cn, Verification Code: E117-48EA-1E96-4A39-5D8F) for their editorial assistance.

Funding: This work was supported by grants from Beijing Science and Technology Planning Project $(\mathrm{CN})$ (grant numbers D17110002617002), National Key Research and Development Program of China (grant numbers 2016YFC1302801), National Key Research and Development Program of China (grant numbers 2018YFC1313103), CAMS Innovation Fund for Medical Sciences (CIFMS) (grant numbers 2016-I2M-1-001), CAMS Innovation Fund for Medical Sciences (CIFMS) (grant numbers 2017-I2M-1-106), CAMS Innovation Fund for Medical Sciences (CIFMS) (grant numbers 2019I2M-2-004), Sanming Project of Medicine in Shenzhen (grant numbers No. SZSM201911008), PUMC Youth Fund and the Fundamental Research Funds for the Central Universities (grant numbers 2017320012).

\section{Footnote}

Reporting Checklist: The authors have completed the STROBE reporting checklist (available at http://dx.doi. org/10.21037/atm-20-2064)

Data Sharing Statement: Available at http://dx.doi. org/10.21037/atm-20-2064

Peer Review File: Available at http://dx.doi.org/10.21037/ atm-20-2064

Conflicts of Interest: All authors have completed the ICMJE uniform disclosure form (available at http://dx.doi. org/10.21037/atm-20-2064). The authors have no conflicts of interest to declare.

Ethical Statement: The authors are accountable for all aspects of the work in ensuring that questions related to the accuracy or integrity of any part of the work are appropriately investigated and resolved. Surgical procedures were approved by the Department of Endoscopy at National Cancer Center/Cancer Hospital, Chinese Academy of Medical Sciences (CICAMS), and the Ethics Committee of National Cancer Center/Cancer Hospital, Chinese Academy of Medical Science and Peking Union Medical College (Approval Number: 18-002/1466). The study was conducted in accordance with the Declaration of Helsinki (as revised in 2013). Written informed consents were obtained from the patient for the surgery and for publication of this cohort study and any accompanying images.

Open Access Statement: This is an Open Access article distributed in accordance with the Creative Commons Attribution-NonCommercial-NoDerivs 4.0 International License (CC BY-NC-ND 4.0), which permits the noncommercial replication and distribution of the article with the strict proviso that no changes or edits are made and the original work is properly cited (including links to both the formal publication through the relevant DOI and the license). See: https://creativecommons.org/licenses/by-nc-nd/4.0/.

\section{References}

1. Torre LA, Bray F, Siegel RL, et al. Global cancer statistics, 2012. CA Cancer J Clin 2015;65:87-108.

2. Bray F, Ferlay J, Soerjomataram I, et al. Global cancer statistics 2018: GLOBOCAN estimates of incidence and mortality worldwide for 36 cancers in 185 countries. CA Cancer J Clin 2018;68:394-424.

3. Patel A, Williams N, Parsons N, et al. Risk factors for metachronous adenoma in the residual colon of patients undergoing curative surgery for colorectal cancer. Int J Colorectal Dis 2017;32:1609-16.

4. Levi F, Randimbison L, Blanc-Moya R, et al. High constant incidence of second primary colorectal cancer. Int J Cancer 2013;132:1679-82.

5. Maehata T, Kato M, Ochiai Y, et al. Feasibility of endoscopic submucosal dissection for colorectal neoplasia at anastomotic sites: a retrospective study. Surg Endosc 2020;34:5495-500.

6. Lopez-Kostner F, Fazio VW, Vignali A, et al. Locally recurrent rectal cancer: predictors and success of salvage 


\section{Page 8 of 8}

surgery. Dis Colon Rectum 2001;44:173-8.

7. Sagar PM, Pemberton JH. Surgical management of locally recurrent rectal cancer. Br J Surg 1996;83:293-304.

8. Horii J, Uraoka T, Goto O, et al. Endoscopic submucosal dissection of colorectal neoplasia located on the suture line of anastomosis. Clin J Gastroenterol 2014;7:290-4.

9. Wada M, Kato M, Hirai $Y$, et al. Salvage resection of recurrent polyp after polypectomy on the colorectal anastomosis using endoscopic submucosal dissection. Gastrointest Endosc 2017;85:1305-6.

10. Hoffman A, Manner H, Rey JW, et al. A guide to multimodal endoscopy imaging for gastrointestinal malignancy - an early indicator. Nat Rev Gastroenterol Hepatol 2017;14:421-34.

11. Watanabe T, Itabashi M, Shimada Y, et al. Japanese Society for Cancer of the Colon and Rectum (JSCCR) Guidelines 2014 for treatment of colorectal cancer. Int J Clin Oncol 2015;20:207-39.

12. Jung WB, Yu CS, Lim SB, et al. Anastomotic Recurrence After Curative Resection for Colorectal Cancer. World J Surg 2017;41:285-94.

13. Schlemper RJ, Riddell RH, Kato Y, et al. The Vienna classification of gastrointestinal epithelial neoplasia. Gut 2000;47:251-5.

14. Li M. Kudo's pit pattern classification for colorectal neoplasms: A meta-analysis. World J Gastroenterol 2014;20:12649-56.

15. Sano Y, Tanaka S, Kudo SE, et al. Narrow-band imaging (NBI) magnifying endoscopic classification of colorectal tumors proposed by the Japan NBI Expert Team. Dig Endosc 2016;28:526-33.

16. Shigita K, Oka S, Tanaka S, et al. Long-term outcomes after endoscopic submucosal dissection for superficial colorectal tumors. Gastrointest Endosc 2017;85:546-53.

17. Barakat M, Seif M, Abdelfatah MM, et al. Endoscopic submucosal dissection for early neoplastic lesions in the surgically altered stomach: a systematic review and metaanalysis. Surg Endosc 2019;33:2381-95.

Cite this article as: Chen Z, Dou L, Zhang Y, He S, Liu Y, Lei H, Wang G. Safety and efficacy of endoscopic submucosal dissection for metachronous early cancer or precancerous lesions emerging at the anastomotic site after curative surgical resection of colorectal cancer. Ann Transl Med 2020;8(21):1411. doi: $10.21037 / \mathrm{atm}-20-2064$
Chen et al. ESD for MECPL emerging at the AS after CRC surgery

18. Nonaka S, Oda I, Makazu M, et al. Endoscopic submucosal dissection for early gastric cancer in the remnant stomach after gastrectomy. Gastrointest Endosc 2013;78:63-72.

19. Nonaka S, Saito Y, Takisawa H, et al. Safety of carbon dioxide insufflation for upper gastrointestinal tract endoscopic treatment of patients under deep sedation. Surg Endosc 2010;24:1638-45.

20. Ojima T, Takifuji K, Nakamura M, et al. Endoscopic submucosal dissection for gastric tumors in various types of remnant stomach. Endoscopy 2014;46:645-9.

21. Kuroki Y, Hoteya S, Mitani T, et al. Endoscopic submucosal dissection for residual/locally recurrent lesions after endoscopic therapy for colorectal tumors. J Gastroenterol Hepatol 2010;25:1747-53.

22. Sakamoto T, Saito Y, Matsuda T, et al. Treatment strategy for recurrent or residual colorectal tumors after endoscopic resection. Surg Endosc 2011;25:255-60.

23. Yamamoto K, Michida T, Nishida T, et al. Colorectal endoscopic submucosal dissection: Recent technical advances for safe and successful procedures. World J Gastrointest Endosc 2015;7:1114-28.

24. Hayashi Y, Miura Y, Yamamoto H. Pocket-creation method for the safe, reliable, and efficient endoscopic submucosal dissection of colorectal lateral spreading tumors. Dig Endosc 2015;27:534-5.

25. Yabuuchi Y, Kakushima N, Takizawa K, et al. Short- and long-term outcomes of endoscopic submucosal dissection for early gastric cancer in the remnant stomach after gastrectomy. J Gastroenterol 2019;54:511-20.

26. Lee JY, Min B-H, Lee JG, et al. Endoscopic Submucosal Dissection for Early Gastric Neoplasia Occurring in the Remnant Stomach after Distal Gastrectomy. Clin Endosc 2016;49:182-6.

27. Tanaka S, Toyonaga T, Morita Y, et al. Endoscopic submucosal dissection for early gastric cancer in anastomosis site after distal gastrectomy. Gastric Cancer 2014;17:371-6. 